Case Report

\title{
Infectious Aortitis: A Life-Threatening Endovascular Complication of Nontyphoidal Salmonella Bacteremia
}

\author{
Seifeldin Hakim (D), ${ }^{1}$ Francisco Davila, ${ }^{1,2}$ Mitual Amin, ${ }^{1,2}$ Ismail Hader, ${ }^{1,2}$ \\ and Mitchell S. Cappell (ii) ${ }^{1,2}$ \\ ${ }^{1}$ Department of Medicine, Division of Gastroenterology, William Beaumont Hospital, Royal Oak, MI 48073, USA \\ ${ }^{2}$ Oakland University William Beaumont School of Medicine, Royal Oak, MI 48073, USA
}

Correspondence should be addressed to Mitchell S. Cappell; mitchell.cappell@beaumont.edu

Received 19 November 2017; Revised 18 February 2018; Accepted 22 February 2018; Published 1 April 2018

Academic Editor: Simon Ching-Shun Kao

Copyright (c) 2018 Seifeldin Hakim et al. This is an open access article distributed under the Creative Commons Attribution License, which permits unrestricted use, distribution, and reproduction in any medium, provided the original work is properly cited.

A 65-year-old Japanese man living in the United States presented with pyrexia and chills associated with intermittent lower abdominal and back pain for 5 days. He denied recent travel, rash, diarrhea, or rectal bleeding. Physical examination revealed spiking pyrexia, and routine laboratory tests revealed mild leukocytosis and neutrophilia. Abdominal CT with contrast showed findings highly compatible with aortitis. Comprehensive autoimmune evaluation was negative. Salmonella enterica serotype Enteritidis was isolated from blood cultures. IV antibiotics were administered, but the patient continued to experience low-grade pyrexia and mild leukocytosis, and follow-up abdominal CT showed progressive aortic inflammation. The patient therefore underwent resection of the affected aortic segment with in-situ graft replacement and lifelong suppressive antibiotics. The patient is asymptomatic with no complications at 18 weeks of follow-up. This case report illustrates that patients with infectious aortitis from nontyphoidal Salmonella may (1) present with nonspecific and nonlocalizing symptoms and signs except for sepsis; (2) have diagnostic blood cultures and abdominal CT findings; and (3) typically require aggressive, prolonged IV antibiotic therapy and surgery for potential cure of this life-threatening infection.

\section{Introduction}

A case is reported of nontyphoidal Salmonella (NTS) causing aortitis and aneurysmal formation, which illustrates that patients with this infection can (1) present with nonspecific and nonlocalizing symptoms of sepsis; (2) have diagnostic blood cultures and abdominal CT findings, and (3) typically require aggressive, prolonged IV antibiotic therapy and surgery for potential cure of this life-threatening infection.

\section{Case Presentation}

A 65-year-old Japanese man with past medical history of hypertension and chronic hepatitis $\mathrm{B}$, and no recent travel history, presented with diffuse body aches, spiking pyrexia, chills, intermittent lower back pain, and abdominal pain unrelated to meals for 5 days. He denied recent diarrhea, rash, or rectal bleeding. Physical examination revealed stable vital signs except temperature $\left(38.9^{\circ} \mathrm{C}\right)$, no jaundice, soft nontender abdomen, guaiac-negative stools, no localized tenderness over the spine, and no limitation of spine mobility. Laboratory analysis revealed a leukocyte count of $10,100 / \mu \mathrm{L}$ (normal: 3,500-10,100/ $\mu \mathrm{L}$ ), neutrophil level of $7,400 / \mu \mathrm{L}$ (normal: 1,600-7,200/ $\mu \mathrm{L}$ ), hemoglobin level of $16.4 \mathrm{~g} / \mathrm{dL}$ (normal: 13.5-17.0 g/dL), platelet count of $266,000 / \mu \mathrm{L}$ (normal: $150,000-400,000 / \mu \mathrm{L}$ ), and serum sodium of $131 \mathrm{mmol} / \mathrm{L}$ (normal: $135-145 \mathrm{mmol} / \mathrm{L}$ ), aspartate aminotransferase of $74 \mathrm{U} / \mathrm{L}$ (normal: 10-37 U/L), alanine aminotransferase of $81 \mathrm{U} / \mathrm{L}$ (normal: 9-47 U/L), and alkaline phosphatase of $101 \mathrm{U} / \mathrm{L}$ (normal: $30-110 \mathrm{U} / \mathrm{L}$ ), with normal total bilirubin and albumin levels. Serum parameters of renal function were within normal limits. The erythrocyte sedimentation rate (ESR) was $107 \mathrm{~mm} /$ hr (normal: $0-15 \mathrm{~mm} / \mathrm{hr}$ ), and the C-reactive protein (CRP) level was $17.4 \mathrm{mg} / \mathrm{dL}$ (normal: $0-0.8 \mathrm{mg} / \mathrm{dL}$ ). He was HIV seronegative, and rapid plasma reagin (RPR) was nonreactive. 

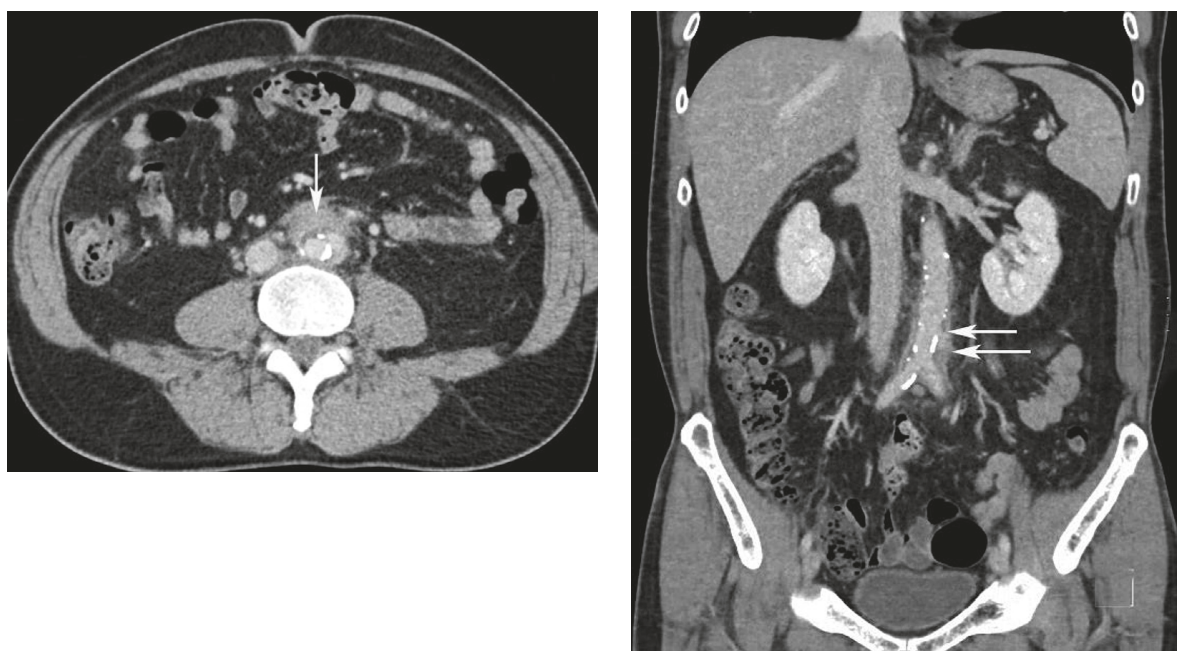

(a)

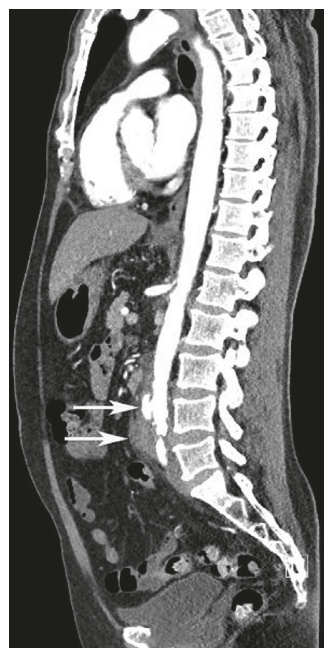

(c) (b)

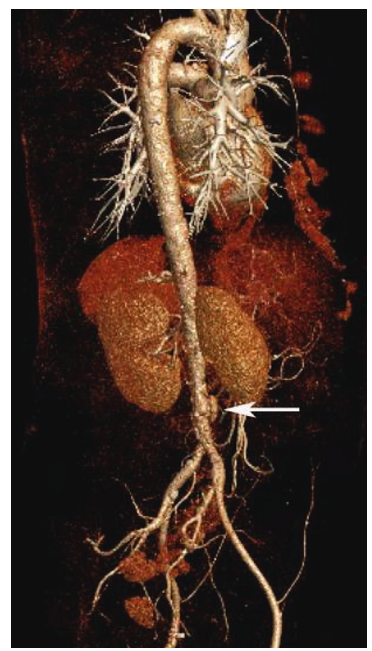

(d)

Figure 1: (a) Transverse view of abdominal CT shows periaortic soft tissue thickening adjacent to aorta (arrow). (b) Coronal view of abdominal CT without IV contrast shows disruption of calcification in aortic wall (upper arrow) and periaortic soft tissue thickening around the infrarenal aorta and around bifurcation of aorta into common iliac arteries (lower arrow). (c) Sagittal view of abdominal CT, with IV contrast, shows pseudoaneurysm formation (upper arrow) and periaortic soft tissue thickening around the infrarenal aorta (lower arrow). (d) Reconstructive CT angiogram shows pseudoaneurysm formation (arrow).

Comprehensive immunological evaluation was within normal limits, including levels/titers of immunoglobulin G subclasses, antinuclear antibodies, rheumatoid factor, cyclic citrullinated peptide, myeloperoxidase, proteinase 3 antibodies, and antineutrophylic cytoplasmic antibodies. The patient was given intravenous (IV) ciprofloxacin $400 \mathrm{mg}$ twice daily empirically in the emergency room before admission.

Abdominal computerized tomography (CT) with intravenous contrast showed inflammatory changes of infrarenal aorta consistent with aortitis. Salmonella enterica serotype Enteritidis was isolated from multiple blood cultures. The bacterial isolate was susceptible to ampicillin, ceftriaxone, and trimethoprim/sulfamethoxazole, but not ciprofloxacin using automated sensitivity testing for 29 antibiotics (NM45, Beckman Coulter Labs., 250 S. Kramer Blvd., Brea, CA 92821).
The antibiotic regimen was switched to IV ceftriaxone $2 \mathrm{~g} / 24 \mathrm{hr}$, but the patient developed progressive abdominal and back pain and persistent pyrexia and mild leukocytosis. Follow-up abdominal CT one week later showed progressive inflammatory changes around infrarenal aorta extending to common iliac arteries and proximal external and internal iliac arteries, mild mural thickening of proximal abdominal aorta, and development of penetrating intimal ulcers, suggesting progression of aortitis (Figures 1(a)-1(d)). He underwent surgical resection of the infected aortic segment with in-situ aortoiliac rifampin-impregnated Dacron graft supported with bilateral iliac arterial stents. Histopathology of the resected aorta revealed severe neutrophylic infiltration and other inflammatory cells within the arterial wall, without evident bacteria (Figure 2). He was discharged after 1 week of IV ceftriaxone therapy to complete a further 5 week 


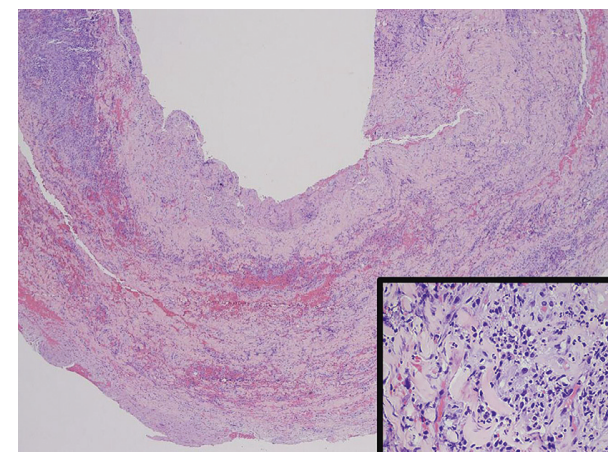

FIGURE 2: Low-power photomicrograph of resected aortic segment with hematoxylin and eosin stain shows inflammation of aortic wall. Inset: high-power photomicrograph of resected aortic segment with hematoxylin and eosin stain shows abundant neutrophylic infiltration of aortic wall without detection of the microorganism.

course of IV therapy as an ambulatory patient. The patient's CRP and ESR were followed weekly after admission. The CRP declined after 1 week to $<0.4 \mathrm{mg} / \mathrm{dL}$ and consistently remained at this low level thereafter. The ESR declined progressively by about $20 \mathrm{~mm} / \mathrm{hr}$ every week until reaching a steady-state level of about $22 \mathrm{~mm} / \mathrm{hr}$ after 4 weeks. He is asymptomatic at 18 -weeks follow-up with planned lifelong antibiotic suppressive therapy with amoxicillin.

\section{Discussion}

Infectious aortitis is a rare, life-threatening, inflammatory process within the aortic wall caused by microorganisms that can lead to aortic aneurysm and rupture [1]. 2.6\% of abdominal aortic aneurysms are secondary to infectious aortitis [2]. Any microorganisms, such as bacteria or fungi, can cause infectious aortitis. In the pre-antibiotic era, syphilis and bacterial endocarditis were the most common infectious etiologies. Currently, common infectious organisms include Gram-positive bacteria such as Staphylococcal species, Enterococcus, or Streptococcus or Gram-negative bacteria such as Salmonella. Uncommon organisms in developed countries are Mycobacterium tuberculosis and Treponema pallidum, but the latter organisms are more common in developing countries. Fungi, such as Candida and Aspergillus, can also infect the aorta [3]. In general, healthy aortic intima is highly resistant to infection, and the major risk factor for aortic infection is atherosclerosis. Mechanisms of aortic infection include (1) most commonly, bacterial seeding of atherosclerotic plaques, (2) extension from contiguous infection, (3) penetrating trauma with direct microorganism invasion of aorta, (4) closed trauma with disruption of aortic intima which may facilitate seeding with microorganisms, (5) septic emboli of aortic vasa vasorum, and (6) self-inflicted or iatrogenic vascular manipulation $[4,5]$.

Salmonella is a Gram-negative, facultative anaerobic, motile, non-lactose-fermenting, and non-spore-forming bacillus [6]. NTS are ubiquitous in nature and infect poultry and reptiles [5]. Salmonella infection can lead to
(1) gastroenteritis, (2) enteric fever, (3) bacteremia, (4) extraintestinal focal infection (EFI), (5) chronic carrier state, and (6) occasionally as bacteremia without gastrointestinal involvement that may lead to EFIs [5, 7]. Salmonella aortitis comprises about $40 \%$ of all infective aortitis $[8,9]$. NTS, especially Salmonella choleraesuis and typhimurium, comprise one-third of abdominal aortic infections [3]. NTS aortitis has a predilection to infect the abdominal aorta, especially infrarenal segment [2]. Bacterial and leukocytic enzymes can further weaken the elastic fibers of the damaged aortic wall leading to aneurysmal formation [3].

Approximately 5\% of patients with NTS gastroenteritis develop bacteremia [10]. EFIs of NTS can infect endovascular tissue, bone, brain, meninges, lungs, or abdominal viscera [10]. The incidence of EFI in NTS bacteremia is $40 \%$ [7]. Endovascular infection is a life-threatening extraintestinal complication of NTS bacteremia. About 25\% of patients $>50$ years old with NTS bacteremia develop endovascular infections. Risk factors for vascular infection in a study of 358 patients $>50$ years old with NTS bacteremia included male sex, hypertension, coronary artery disease, and serogroup $\mathrm{C} 1$ infections, whereas negative risk factors include malignancy and immunosuppressive therapy [11]. Although immunosuppression predisposes subjects to NTS bacteremia, immunosuppression may counteract formation of endovascular infections [12].

Symptoms are variable, but may include the following: (1) gastroenteritis: diarrhea, nausea, vomiting, and abdominal cramps; (2) infectious symptoms: pyrexia, chills, and sweats; (3) aortitis: location of the involved aortic segment determines the type and severity of symptoms: abdominal pain if abdominal aorta is affected; chest, shoulder, or back pain if thoracic aorta is affected; and shock or hemodynamic instability if an aneurysm forms and ruptures; and (4) other symptoms of EFIs: endocarditis, septic arthritis, osteomyelitis, cholangitis, meningitis, pneumonia, or other manifestations of visceral organ involvement $[2,10]$.

Diagnosis of Salmonella aortitis requires a high index of suspicion in the presence of risk factors, NTS bacteremia, and compatible symptoms even in hemodynamically stable patients. Chen et al. [11] reported in 2012 a scoring system for NTS vascular infections (NTSVIs) to help predict the risk of vascular infections in patients with NTS bacteremia. In this scoring system, each of the following is assigned 1 point: male sex, hypertension, coronary artery disease, and serogroup $\mathrm{C} 1$ infection; and each of the following is assigned -1 point: malignancy, and immunosuppression due to negative association with aortitis. Based on this scoring system, the prevalence of vascular infections in patients with $0,1,2,3$, or 4 points was $2.2 \%, 10.6 \%, 39.4 \%$, $55.2 \%$, and $100 \%$, respectively $[11,12]$.

Supporting data include laboratory studies showing leukocytosis and neutrophilia, elevated markers of inflammation such as ESR or CRP, and positive blood cultures $[2,7]$. Pretreatment with antibiotics can lead to absence of microorganisms in the resected aortic specimen, as occurred in this case, and sterile blood cultures [4, 13].

Abdominal CT with IV contrast is the diagnostic study of choice. However, CT can show normal aorta during early 
aortitis, as it may miss the initial changes in the arterial wall or periaortic tissue [14]. Diagnostic CT findings of aortic inflammation include mural thickening, periaortic soft tissue density, rim enhancement, periaortic gas, periaortic stranding or fluid retention, saccular or fusiform aneurysm, disruption of calcification, and vertebral body erosion $[15,16]$. Magnetic resonance imaging (MRI) with gadolinium enhancement is an emerging imaging technique that may have similar diagnostic potential as CT, but should not be used in patients with implanted devices or unstable patients [4]. Digital subtraction angiography (DSA) and invasive aortography are reserved for cases in which the diagnosis of acute aortitis cannot be excluded by noninvasive methods or to confirm the diagnosis. DSA and aortography have the disadvantage of imaging the aortic lumen only without showing adjacent soft tissue or bony involvement. Invasive aortography carries the additional risk of rupture of the fragile aortic wall from increased intraprocedural aortic pressure $[4,15]$.

NTS aortitis is usually treated by combined medical and surgical therapy. The mortality is $96-100 \%$ with medical therapy alone and is $40 \%$ with combined medical and surgical therapy [2]. Initial empiric antibiotic therapy for infectious aortitis should generally cover Gram-positive and Gram-negative bacteria, taking into consideration risk factors for multidrug-resistant organisms and/or extended spectrum beta-lactamase-resistant bacteria that may require specific antibiotics (e.g., vancomycin, ceftaroline, daptomycin, linezolid, carbapenem, or newer agents) [17, 18]. When Salmonella aortitis is confirmed, beta-lactam antibiotics (ceftriaxone, piperacillin-tazobactam, or other betalactams) are preferred if the bacteria are susceptible to them because some emerging Salmonella strains, especially in Asia, have increasing resistance to ciprofloxacin and other conventional antibiotic therapies [19]. If surgery is not urgently indicated (neither impending aortic rupture nor hemodynamic instability), intravenous antibiotics should be administered for 2-4 weeks before surgery to control the local infection to prevent reinfection, especially for in-situ graft placement. Intravenous antibiotics should be extended postoperatively until 6-12 weeks after blood cultures become sterile $[2,13]$.

The optimal duration of antibiotic therapy remains controversial, with some experts suggesting lifelong suppressive oral antibiotic therapy after IV antibiotics especially for difficult to treat microorganisms or for in-situ graft placement [20]. Long-term suppressive antibiotic therapy with oral agents depends on culture susceptibility, but the data are limited about specific antibiotics, antibiotic dosages, and duration of therapy [21-23]. Most studies of antibiotic therapies included ciprofloxacin, amoxicillin, amoxicillinclavulanic acid, or trimethoprim-sulfamethoxazole antibiotic agents.

The standard surgical treatment is resection of the infected aortic segment and in-situ or extra-anatomical reconstruction. Theoretically, in-situ graft is more prone to graft reinfection, whereas extra-anatomical graft is more prone to thrombosis [4]. Endovascular aortic repair (EVAR) can be used as a temporary measure to hemodynamically stabilize unstable patients or to seal vascular leaks until the patient undergoes definitive surgery. EVAR has been used as a stand-alone technique to surgically repair mycotic aortic aneurysms and Salmonella aortic aneurysms, but the current data on efficacy are inadequate [3, 4, 10, 23, 24]. Study limitations include this is a single case report, and it is retrospectively reported.

\section{Conclusion}

A case is reported of NTS causing aortitis and aneurysmal formation. The patient presented nonspecifically with diffuse body aches, spiking pyrexia, chills, intermittent lower back pain, and abdominal pain unrelated to meals for 5 days, without recent diarrhea, rash, or rectal bleeding. Blood cultures and abdominal CT findings were diagnostic. The patient was successfully treated with prolonged IV antibiotic therapy, segmental aortic resection, and graft repair. This case illustrates the potential elusiveness of this diagnosis.

NTS aortitis is a life-threatening disease associated with up to $100 \%$ mortality if inadequately treated. Early diagnosis is crucial to improve outcome. However, the diagnosis can be challenging as blood cultures can be sterile due to prior antibiotic use, CT scan can be normal early in the infection, and other diseases can lead to aortitis. Diagnosis of NTS aortitis requires awareness of risk factors, recognition of symptoms, and a high index of suspicion. CT scan is the imaging modality of choice. Early diagnosis, early use of appropriate antibiotics, and definitive surgical repair may reduce mortality to $40 \%$.

\section{Ethical Approval}

The IRB at William Beaumont Hospital, Royal Oak, approved/ exempted this study.

\section{Disclosure}

A preliminary announcement of this study was presented as an abstract/poster at the 2017 meeting of the American College of Gastroenterology on October 17, 2017: Seifeldin Hakim, Mitual Amin, Ismail Hader, and Mitchell S. Cappell, "Salmonella aortitis: A rare life-threatening complication of non-typhoid Salmonella bacteremia," Program (poster) No. P2464, World Congress of Gastroenterology at ACG 2017 Meeting Abstracts, American College of Gastroenterology, Orlando, FL. Dr. Cappell, as a consultant of the United States Food and Drug Administration (FDA) Advisory Committee for Gastrointestinal Drugs, affirms that this paper does not discuss any proprietary confidential pharmaceutical data submitted to the FDA. Dr. Cappell is also a member of the speaker's bureau for AstraZeneca and Daiichi Sankyo, comarketers of Movantik, and received a one-time consulting fee from Mallinckrodt. This work does not discuss any drug manufactured or marketed by AstraZeneca, Daiichi Sankyo, or Mallinckrodt.

\section{Conflicts of Interest}

The authors declare that they have no conflicts of interest. 


\section{Authors' Contributions}

Seifeldin Hakim and Mitchell S. Cappell contributed equally as primary authors to this work.

\section{References}

[1] M. Nakayama, K. Fuse, M. Sato et al., "Infectious aortitis caused by Salmonella Dublin followed by aneurysmal dilatation of the abdominal aorta," Internal Medicine, vol. 51, no. 20, pp. 2909-2911, 2012.

[2] V. A. Soravia-Dunand, V. G. Loo, and I. E. Salit, "Aortitis due to Salmonella: report of 10 cases and comprehensive review of the literature," Clinical Infectious Diseases, vol. 29, no. 4, pp. 862-868, 1999.

[3] E. A. Foote, R. G. Postier, R. A. Greenfield, and M. S. Bronze, "Infectious aortitis," Current Treatment Options in Cardiovascular Medicine, vol. 7, no. 2, pp. 89-97, 2005.

[4] R. J. Lopes, J. Almeida, P. J. Dias, P. Pinho, and M. J. Maciel, "Infectious thoracic aortitis: a literature review," Clinical Cardiology, vol. 32, no. 9, pp. 488-490, 2009.

[5] L. A. Salzberger, D. Cavuoti, and J. Barnard, "Fatal Salmonella aortitis with mycotic aneurysm rupture," American Journal of Forensic Medicine and Pathology, vol. 23, no. 4, pp. 382-385, 2002.

[6] J. Kedzierska, B. Piatkowska-Jakubas, A. Kedzierska et al., "Clinical presentation of extraintestinal infections caused by non-typhoid Salmonella serotypes among patients at the University Hospital in Cracow during an 7-year period," Polish Journal of Microbiology, vol. 57, no. 1, pp. 41-47, 2008.

[7] P. L. Chen, C. M. Chang, C. J. Wu et al., "Extraintestinal focal infections in adults with nontyphoid Salmonella bacteraemia: predisposing factors and clinical outcome," Journal of Internal Medicine, vol. 261, no. 1, pp. 91-100, 2007.

[8] N. Tarif, A. El Kheder Ael, M. N. Azam, A. H. Mitwalli, and J. S. Al-Wakeel, "Non-typhoidal Salmonella aortitis in a transplant patient," Saudi Journal of Kidney Diseases and Transplantation, vol. 13, no. 4, pp. 501-505, 2002.

[9] P. S. Cohen, T. F. O’Brien, S. C. Schoenbaum, and A. A. Medeiros, "The risk of endothelial infection in adults with Salmonella bacteremia," Annals of Internal Medicine, vol. 89, no. 6, pp. 931-932, 1978.

[10] M. L. Fernández Guerrero, J. M. Aguado, A. Arribas, C. Lumbreras, and M. de Gorgolas, "The spectrum of cardiovascular infections due to Salmonella enterica: a review of clinical features and factors determining outcome," Medicine, vol. 83, no. 2, pp. 123-138, 2004.

[11] P. L. Chen, C. C. Lee, C. Y. Li et al., "A simple scoring algorithm predicting vascular infections in adults with nontyphoid Salmonella bacteremia," Clinical Infectious Diseases, vol. 55, no. 2, pp. 194-200, 2012.

[12] A. Dhanoa and Q. K. Fatt, "Non-typhoidal Salmonella bacteraemia: epidemiology, clinical characteristics and its' association with severe immunosuppression," Annals of Clinical Microbiology and Antimicrobials, vol. 8, p. 15, 2009.

[13] H. L. Gornik and M. A. Creager, "Aortitis," Circulation, vol. 117, no. 23, pp. 3039-3051, 2008.

[14] C. T. Stephens, L. L. Pounds, and L. A. Killewich, "Rupture of a nonaneurysmal aorta secondary to Staphylococcus aortitis," Angiology, vol. 57, no. 4, pp. 506-512, 2006.

[15] M. P. Lin, S. C. Chang, R. H. Wu, C. K. Chou, and W. S. Tzeng, "A comparison of computed tomography, magnetic resonance imaging, and digital subtraction angiography findings in the diagnosis of infected aortic aneurysm," Journal of
Computer Assisted Tomography, vol. 32, no. 4, pp. 616-620, 2008.

[16] T. A. Macedo, A. W. Stanson, G. S. Oderich, C. M. Johnson, J. M. Panneton, and M. L. Tie, "Infected aortic aneurysms: imaging findings," Radiology, vol. 231, no. 1, pp. 250-257, 2004.

[17] S. S. Jean, Y. T. Lee, S. M. Guo, and P. R. Hsueh, "Recurrent infections caused by cefotaxime- and ciprofloxacin-resistant Salmonella enterica serotype choleraesuis treated successfully with imipenem," Journal of Infection, vol. 51, no. 3, pp. e163-e165, 2005.

[18] R. M. Humphries, F. C. Fang, F. M. Aarestrup, and J. A. Hindler, "In vitro susceptibility testing of fluoroquinolone activity against Salmonella: recent changes to CLSI standards," Clinical Infectious Diseases, vol. 55, no. 8, pp. 1107-1113, 2012.

[19] D. A. Williamson, C. R. Lane, M. Easton et al., "Increasing antimicrobial resistance in nontyphoidal Salmonella isolates in Australia from 1979 to 2015," Antimicrobial Agents and Chemotherapy, vol. 62, no. 2, pp. e02012-e02017, 2018.

[20] A. C. Ting, S. W. Cheng, P. Ho, J. T. Poon, and J. H. Tsu, "Surgical treatment of infected aneurysms and pseudoaneurysms of the thoracic and abdominal aorta," American Journal of Surgery, vol. 189, no. 2, pp. 150-154, 2005.

[21] C. M. Luo, C. Y. Chan, Y. S. Chen, S. S. Wang, N. H. Chi, and I. H. Wu, "Long-term outcome of endovascular treatment for mycotic aortic aneurysm," European Journal of Vascular and Endovascular Surgery, vol. 54, no. 4, pp. 464-471, 2017.

[22] S. Pulimamidi, F. J. Caputo, H. S. Fraimow, R. Nahra, and C. Tsigrelis, "Salmonella aortitis treated with endovascular aortic repair," Annals of Vascular Surgery, vol. 28, no. 5, pp. 1314.e5-1314.e10, 2014.

[23] B. Kritpracha, D. Premprabha, J. Sungsiri, W. Tantarattanapong, S. Rookkapan, and P. Juntarapatin, "Endovascular therapy for infected aortic aneurysms," Journal of Vascular Surgery, vol. 54, no. 5, pp. 1259-1265, 2011.

[24] P. J. Parekh, R. Shams, V. Challapallisri, and P. E. Marik, "Successful treatment of Salmonella aortitis with endovascular aortic repair and antibiotic therapy," BMJ Case Reports, vol. 2014, no. 1, 2014. 


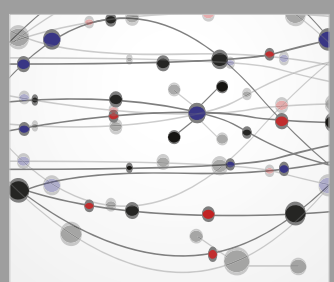

The Scientific World Journal
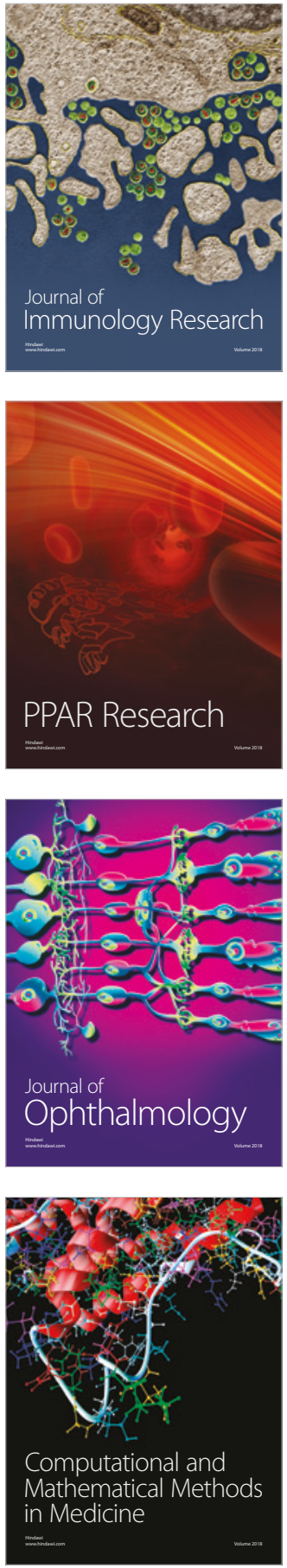

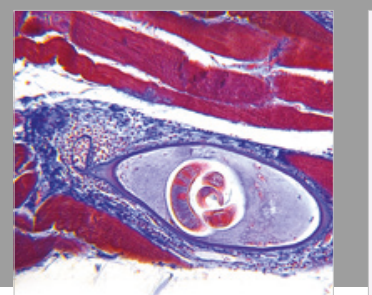

Gastroenterology Research and Practice

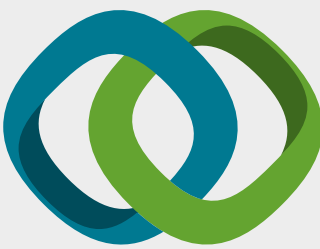

\section{Hindawi}

Submit your manuscripts at

www.hindawi.com
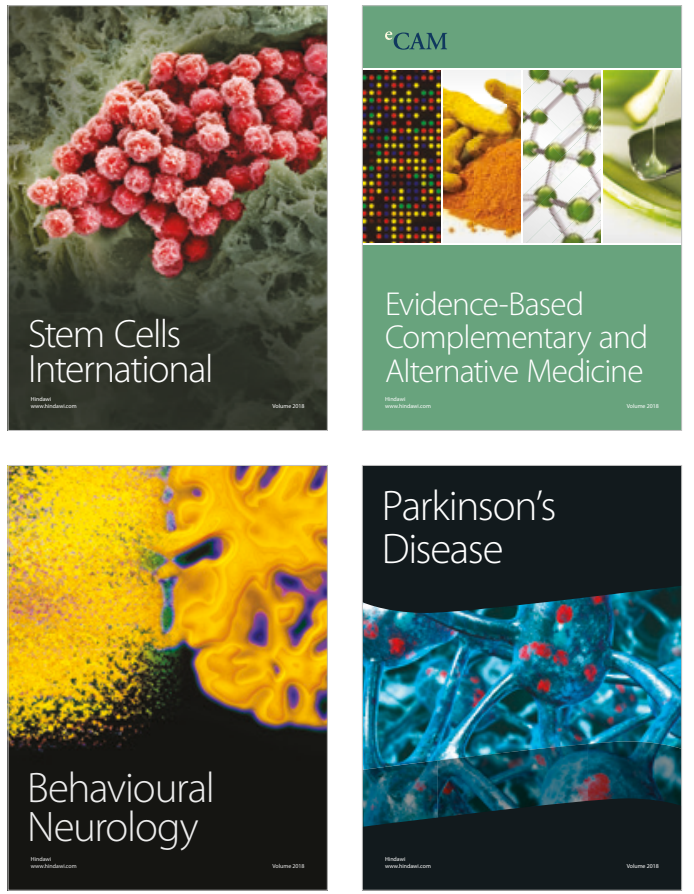

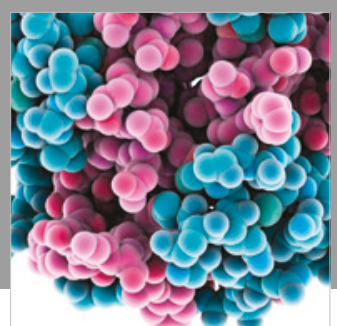

ournal of

Diabetes Research

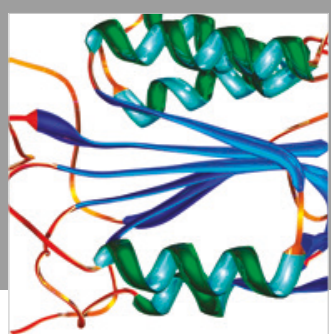

Disease Markers
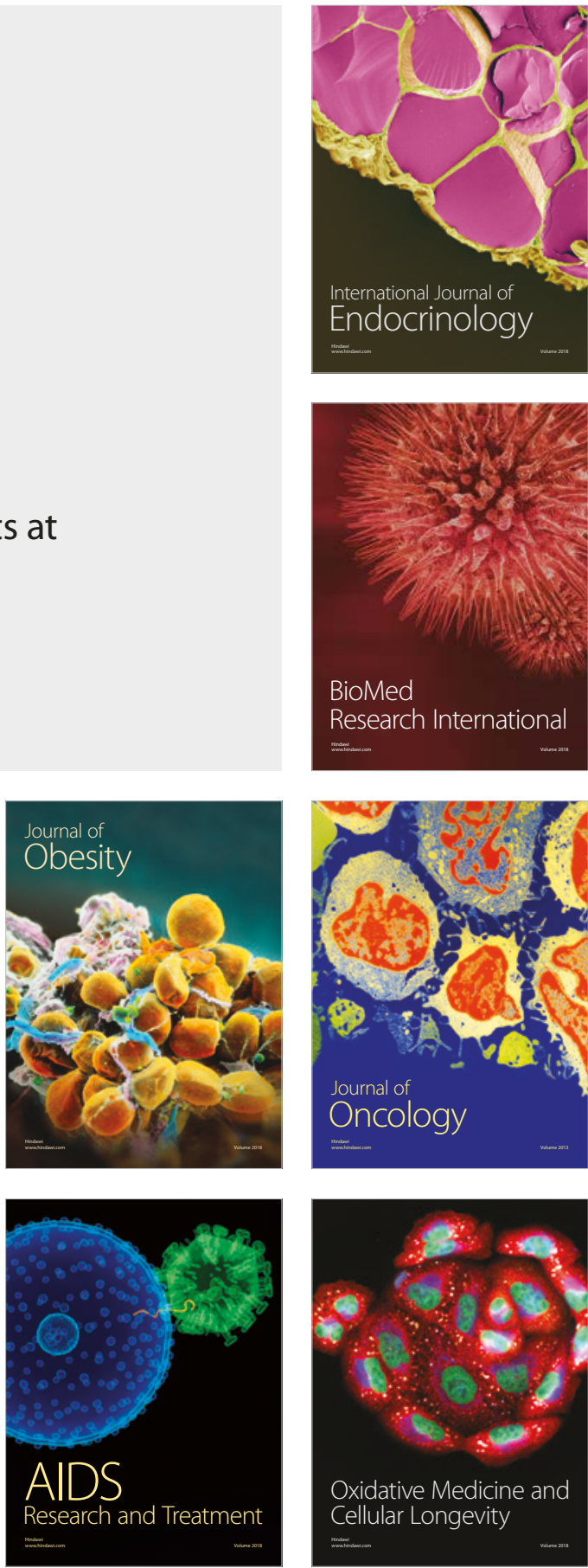